Also available at http://amc.imfm.si ISSN 1855-3966 (printed edn.), ISSN 1855-3974 (electronic edn.)

ARS MATHEMATICA CONTEMPORANEA 5 (2012) 223-234

\title{
Minimum cycle bases of lexicographic products
}

\author{
Marc Hellmuth \\ Center for Bioinformatics, Saarland University \\ Building E 2.1, D-66041 Saarbrücken, Germany,

\section{Philipp-Jens Ostermeier} \\ Max Planck Institute for Mathematics in the Sciences \\ Inselstraße 22, D-04103 Leipzig, Germany
}

\section{Peter F. Stadler}

Bioinformatics Group, Department of Computer Science and Interdisciplinary Center of Bioinformatics, University of Leipzig

Härtelstraße 16-18, D-04107 Leipzig, Germany

Max Planck Institute for Mathematics in the Sciences Inselstraße 22, D-04103 Leipzig, Germany

Fraunhofer Institut f. Zelltherapie und Immunologie Perlickstraße 1, D-04103 Leipzig, Germany

Inst. f. Theoretical Chemistry, University of Vienna

Währingerstraße 17, A-1090 Wien, Austria

Santa Fe Institute, 1399 Hyde Park Rd., Santa Fe, NM 87501, USA

Received 6 September 2010, accepted 2 January 2012, published online 22 March 2012

\begin{abstract}
Minimum cycle bases of product graphs can in most situations be constructed from minimum cycle bases of the factors together with a suitable collection of triangles and/or quadrangles determined by the product operation. Here we give an explicit construction for the lexicographic product $G \circ H$ that generalizes results by Berger and Jaradat to the case that $H$ is not connected.
\end{abstract}

Keywords: Cycle space, cycle basis, lexicographic product.

Math. Subj. Class.: 05C10

E-mail addresses: marc.hellmuth@bioinf.uni-sb.de (Marc Hellmuth), Philipp.Ostermeier@mis.mpg.de (Philipp-Jens Ostermeier), studla@bioinf.uni-leipzig.de (Peter F. Stadler) 


\section{Introduction}

Throughout this contribution, let $G=(V, E)$ be a finite undirected simple graph. A (generalized) cycle in $G$ is an Eulerian subgraph of $G$, i.e., a subgraph of $G$ in which the degree of every vertex is even. A connected Eulerian subgraph in which every vertex has degree 2 will be called an elementary cycle.

The cycle space $\mathcal{C}(G)$ of a graph $G$ is the subspace of the edge space generated by the elementary cycles. Hence we regard cycles as subsets of $E(G)$. A cycle basis is a basis of $\mathcal{C}(G)$. If $G$ has $c(G)$ connected components, $\mathcal{C}(G)$ has dimension $\beta(G)=|E(G)|-$ $|V(G)|+c(G)$. An minimum cycle basis (MCB) is a cycle basis $\mathcal{B}$ that minimizes the total length

$$
\ell(\mathcal{B})=\sum_{C \in \mathcal{B}}|E(C)|
$$

of the basis cycles. MCBs play a role e.g. in the analysis of electrical circuits, periodic scheduling problems in traffic planning, graph drawing [18, 14], mechanical frame analysis [13], biopolymer structures [17, 4, 16], and computational chemistry [2, 15].

Four standard graph products have received considerable attention [9, 8]: the Cartesian, direct, strong and lexicographic product of graphs. MCBs of these product graphs can in most cases be constructed from MCBs of their factors and a suitable collection of short cycles that can quite easily be specified explicitly. A complete characterization for the Cartesian and the strong product can be found in [10]. The situation is more complex for the direct product of simple graphs. Constructions for MCBs are known for bipartite factors [5], products of complete graphs [6,3], and graphs of the form $G \times C_{q}$ where $G$ is connected non-bipartite and $C_{q}$ is an odd cycle [7]. MCBs for the lexicographic product $G \circ H$ consist of a single copy of the MCB of $G$ and suitable set of triangles provided $H$ is connected $[1,11]$. Here, we derive an MCB for lexicographic products $G \circ H$ with a disconnected 2nd factor by combining the Berger-Jaradat construction for connected $H$ with an MCB of $G \circ \bar{K}_{n}$, where $\bar{K}_{n}$ is the graph on $n$ vertices without edges.

The lexicographic product $G \circ H$ has the vertex set $V(G \circ H)=V(G) \times V(H)$. There is an edge $\{(a, x),(b, y)\} \in E(G \circ H)$ if $\{a, b\} \in E(G)$ or $a=b$ and $\{x, y\} \in$ $E(H)$. The connectivity of $G \circ H$ is therefore determined by the connectivity of $G$ unless $G=K_{1}$. More precisely, let $G_{i}, i \in I$ be the connected components of $G$ and assume $G_{i} \neq K_{1}$. Then the connected components of $G \circ H$ are the lexicographic products $G_{i} \circ$ $H$, irrespective of whether $H$ is connected or not. Since $K_{1} \circ H=H$, the connected components are determined by $H$ in this case. For convenience, we write $x \circ H$ and $a b \circ H$ for the lexicographic product of $H$ with the $K_{1}$ consisting of the single vertex $x$ and the $K_{2}$ consisting of the single edge $\{a, b\}$, respectively. The $G^{x}$-layer (or $G^{x}$-fiber) is the subgraph of $G \circ H$ induced by the vertices of the form $(a, x), a \in V(G)$. Analogously, the $H^{a}$-layer is the subgraph induced by the vertices of the form $(a, x), x \in V(H)$. We will write $\mathcal{B}$ for a cycle basis of $G$ and $\mathcal{B}^{x}$ denotes the copy of $\mathcal{B}$ within the $G^{x}$-layer. The set $\{v \in V(G) \mid\{a, v\} \in E(G)\}$ of all neighbors of a vertex $a$ is denoted by $N(a)$.

Remark 1.1. Since the cycle space of a graph is the direct sum of the cycle spaces of its connected components we can assume in the following that without loss of generality $G$ is connected and is distinct from $K_{1}$.

In order to determine whether a given cycle basis is an MCB we use the following well-known criterion, see e.g. [7]. 
Lemma 1.2. A cycle basis $\mathcal{B}$ for a graph $G$ is an $M C B$ if and only if every cycle $C \in \mathcal{C}(G)$ is a sum of basis elements whose lengths do not exceed $|E(C)|$.

\section{MCB of $G \circ \bar{K}_{n}$}

In order to show how to construct MCBs of products of disconnected graphs, we are in this section concerned with the special simpler instance $G \circ \bar{K}_{n}$. Let us first consider the case that $G$ is triangle-free. In this case $G \circ \bar{K}_{n}$ is also triangle-free. We start with the observation that $K_{2} \circ \bar{K}_{n}=K_{n, n}$. As shown by Kainen [12], $K_{n, n}$ has an MCB $\mathcal{K}$ consisting entirely of quadrangles. Setting $V\left(K_{2}\right)=\{a, b\}$ and fixing a vertex $x_{0} \in$ $V\left(\bar{K}_{n}\right)$ the $(n-1)^{2}$ quadrangles of $\mathcal{K}$ are of the form $\left\langle\left(a, x_{0}\right)\left(b, x_{0}\right)(a, u)(b, v)\right\rangle$ with $u, v \neq x_{0}$. For arbitrary graphs $G$ and a fixed edge $\{a, b\} \in E(G)$ we will denote the elements $\left\langle\left(a, x_{0}\right)\left(b, x_{0}\right)(a, u)(b, v)\right\rangle, u, v \neq x_{0}$ of $\mathcal{K}$ by $\mathcal{K}_{a b}$.

Remark 2.1. In the following, we will denote the fixed vertex that determines a particular Kainen basis by $x_{0}$.

Now consider, for each vertex $a \in V(G)$ a set of quadrangles of the form $C_{b c}^{v}:=$ $\left\langle\left(b, x_{0}\right)\left(a, x_{0}\right)\left(c, x_{0}\right)(a, v)\right\rangle$ where $x_{0}$ is again the fixed vertex in $V\left(\bar{K}_{n}\right), b$ and $c$ are neighbors of $a$ in $G$. Clearly, $\{a, b\} \in E(G)$ and $\{a, c\} \in E(G)$, and $v \neq x_{0}$. Not all such combinations of $b$ and $c$ are linearly independent. In particular, we observe that $C_{b c}^{v} \oplus C_{b d}^{v}=C_{c d}^{v}$. Thus, consider the star graph consisting of $a$ and its neighbors $N(a)$ in $G$ and $f i x$ an arbitrary $b \in N(a)$. It follows that

$$
\mathcal{G}_{a}:=\left\{C_{b c}^{v} \mid c \in N(a) \backslash\{b\}, v \in V\left(\bar{K}_{n}\right) \backslash\left\{x_{0}\right\}\right\}
$$

is linearly independent because each cycle contains an edge $\left\{(a, v),\left(c, x_{0}\right)\right\}$, that is not contained in any other cycle of $\mathcal{G}_{a}$.

Lemma 2.2. The cycle set $\mathcal{Q}:=\bigcup_{a b \in E(G)} \mathcal{K}_{a b} \cup \bigcup_{a \in V(G)} \mathcal{G}_{a}$ is linearly independent.

Proof. Since the $|E(G)|$ copies of the $K_{n, n}$ are pairwise edge disjoint we see that the $|E(G)|(n-1)^{2}$ cycles in $\mathcal{K}:=\bigcup_{a b \in E(G)} \mathcal{K}_{a b}$ are linearly independent. Furthermore, since each cycle in $\mathcal{G}_{u}$ contains an edge that is not contained in any other cycle in $\mathcal{G}_{v}, v \neq u$, we can conclude that the $\sum_{a \in V(G)}(\operatorname{deg}(a)-1)=2|E(G)|-|V(G)|$ cycles in $\bigcup_{a \in V(G)} \mathcal{G}_{a}$ are linearly independent.

The symmetric difference of any two distinct quadrangles in $\bigcup_{a b \in E(G)} \mathcal{K}_{a b}$ results in a cycle that contains at least one elementary cycle. Notice that there is at least one elementary cycle that is entirely contained in a subgraph $a b \circ \bar{K}_{n}$ for some edge $\{a, b\} \in E(G)$, since the elements of the Kainen basis of different sets $\mathcal{K}_{u v}$ and $\mathcal{K}_{x y}$ are edge disjoint. W.1.o.g. let $C$ be such a cycle that contains the elementary cycle $c=\left\langle\left(a, s_{1}\right)\left(b, s_{2}\right) \ldots\left(a, s_{2 j-1}\right)\right.$ $\left.\left(b, s_{2 j}\right)\right\rangle$ with $s_{i} \in V\left(\bar{K}_{n}\right), j \leq l$. By construction and since $|c| \geq 4$, we can conclude that $c$ contains an edge $\left\{\left(a, s_{f}\right),\left(b, s_{g}\right)\right\}$ with $s_{f}, s_{g} \neq x_{0}$.

Now, we use elements from $\bigcup_{a \in V(G)} \mathcal{G}_{a}$ for the construction of $c$. We observe that the edge $\left\{\left(a, s_{f}\right),\left(b, s_{g}\right)\right\}$ is not included in any cycle of $\bigcup_{a \in V(G)} \mathcal{G}_{a}$. Hence, the symmetric difference of cycles in $\bigcup_{a \in V(G)} \mathcal{G}_{a}$ never contains an elementary cycle in $a b \circ \bar{K}_{n}$ for any edge $\{a, b\} \in G$. Consequently, $\operatorname{span}\left(\bigcup_{a b \in E(G)} \mathcal{K}_{a b}\right) \cap \operatorname{span}\left(\bigcup_{a \in V(G)} \mathcal{G}_{a}\right)=\varnothing$ and hence, $\mathcal{Q}$ is a linearly independent set. 
Theorem 2.3. Let $\mathcal{B}$ be an $M C B$ of a graph $G$. If $G \neq K_{1}$ is a connected triangle-free graph, then $\mathcal{Q} \cup \mathcal{B}^{x_{0}}$ is an $M C B$ of $G \circ \bar{K}_{n}$.

Proof. We show first that no cycle within the $G^{x_{0}}$-fiber can be generated by elements of $\mathcal{Q}$. Suppose that such a cycle $C$ exists. W.l.o.g. let $\left\{\left(a, x_{0}\right),\left(b, x_{0}\right)\right\}$ and $\left\{\left(b, x_{0}\right),\left(c, x_{0}\right)\right\}$ be two consecutive edges of $C$. In order to generate these edges we can only use squares contained in $\mathcal{K}_{a b}$ and $\mathcal{K}_{b c}$, resp., or squares contained in $\mathcal{G}_{b}, \mathcal{G}_{a}$, and $\mathcal{G}_{c}$.

If we use the square $S_{1}=\left\langle\left(a, x_{0}\right),\left(b, x_{0}\right),\left(a, x_{i}\right),\left(b, x_{j}\right)\right\rangle \in \mathcal{K}_{a b}$ or $S_{2}=\left\langle\left(b, x_{0}\right)\right.$, $\left.\left(c, x_{0}\right),\left(b, x_{l}\right),\left(c, x_{k}\right)\right\rangle \in \mathcal{K}_{b c}$, we must finally remove the edges $\left\{\left(a, x_{i}\right),\left(b, x_{j}\right)\right\}$ and $\left\{\left(b, x_{l}\right),\left(c, x_{k}\right)\right\}$, which is only possible if we compute $S_{1} \oplus S_{1}$ or $S_{2} \oplus S_{2}$, a contradiction.

If we use one of the squares in $\mathcal{G}_{a}, \mathcal{G}_{b}$, or $\mathcal{G}_{c}$, e.g. $\left\langle\left(b, x_{0}\right),\left(c, x_{0}\right),\left(d, x_{0}\right),\left(c, x_{r}\right)\right\rangle \in$ $\mathcal{G}_{c}$, we must remove the edge $\left\{\left(b, x_{0}\right),\left(c, x_{r}\right)\right\}$, which is only possible by using a square $\left\langle\left(b, x_{0}\right),\left(c, x_{0}\right),\left(b, x_{s}\right),\left(c, x_{r}\right)\right\rangle \in \mathcal{K}_{b c}$. By the same arguments as above, it follows that this square must be used twice in order to cancel the remaining edge $\left\{\left(b, x_{s}\right),\left(c, x_{r}\right)\right\}$. The same holds for the other squares contained in $\mathcal{G}_{b}$ and $\mathcal{G}_{a}$. Therefore, we cannot generate a cycle $C$ that is within the $G^{x_{0}}$-layer using elements of $\mathcal{Q}$ and hence, $\mathcal{Q} \cup \mathcal{B}^{x_{0}}$ is linearly independent.

Since $G$ is triangle-free, the cycles of minimal length are quadrangles. $\mathcal{Q}$ consists of

$$
\begin{aligned}
&|\mathcal{Q}|=|E(G)|(n-1)^{2}+\sum_{a \in V(G)}(\operatorname{deg}(a)-1)(n-1)= \\
&|E(G)|(n-1)^{2}+(2|E(G)|-|V(G)|)(n-1)
\end{aligned}
$$

elements. Together with the $\beta(G)$ cycles of $\mathcal{B}^{x_{0}}$ this equals the required number of $\beta(G \circ$ $\left.\bar{K}_{n}\right)=|E(G)| n^{2}-|V(G)| n+1$ basis cycles. Hence, $\mathcal{Q} \cup \mathcal{B}^{x_{0}}$ is a basis.

It remains to show that the constructed basis is minimal. To show this we first observe that by construction $\mathcal{B}^{x_{0}}$ is an MCB of $\operatorname{span}\left(\mathcal{B}^{x_{0}}\right)$ and by Lemma $1.2 \mathcal{Q}$ is an MCB of $\operatorname{span}(\mathcal{Q})$. Since $\mathcal{Q} \cup \mathcal{B}^{x_{0}}$ is a basis no cycle entirely contained in the $x_{0}$-fiber can be constructed as linear combination of $\mathcal{Q}$ and no cycle in $\operatorname{span}(\mathcal{Q})$ is a linear combination of cycles from $\mathcal{B}^{x_{0}}$. As a consequence, $C \in \mathcal{C}\left(G \circ \bar{K}_{n}\right)$ can be written in the form $C=$ $C^{\prime} \oplus C^{\prime \prime}$ where $C^{\prime} \in \operatorname{span}\left(\mathcal{B}^{x_{0}}\right)$ and $C^{\prime \prime} \in \operatorname{span}(\mathcal{Q})$. By construction of $\mathcal{Q}$ we can conclude that the number of edges of $C^{\prime \prime}$ contained in $\left(G \circ \bar{K}_{n}\right) \backslash G^{x_{0}}$ is greater or equal than the number of its edges in $G^{x_{0}}$. More formally, $n_{1}=\mid E\left(C^{\prime \prime}\right) \cap E\left(G \circ \bar{K}_{n}\right) \backslash$ $E\left(G^{x_{0}}\right)|\geq| E\left(C^{\prime \prime}\right) \cap E\left(G^{x_{0}}\right) \mid=n_{2}$ and therefore, $n_{1}-n_{2} \geq 0$. Hence, we have $|E(C)|=\left|E\left(C^{\prime}\right)\right|+n_{1}-n_{2} \geq\left|E\left(C^{\prime}\right)\right|$. Since $\mathcal{B}^{x_{0}}$ is an MCB of $\operatorname{span}\left(\mathcal{B}^{x_{0}}\right)$, we can conclude that $|E(C)| \geq\left|E\left(C^{\prime}\right)\right| \geq\left|E\left(B_{C}\right)\right|$ with $B_{C} \in \mathcal{B}^{x_{0}}$. Moreover, since $G$ is triangle-free and all elements of $\mathcal{Q}$ have length four it holds for every $B_{C} \in \mathcal{B}^{x_{0}}$ and each $Q_{C} \in \mathcal{Q}$ that $|E(C)| \geq\left|E\left(C^{\prime}\right)\right| \geq\left|E\left(B_{C}\right)\right| \geq\left|E\left(Q_{C}\right)\right|$. Therefore, Lemma 1.2 implies that the length of the cycle basis $\mathcal{Q} \cup \mathcal{B}^{x_{0}}$ cannot be reduced by exchanging any of its elements by $C$; hence $\mathcal{Q} \cup \mathcal{B}^{x_{0}}$ is an MCB.

\section{The general case}

Let us now suppose that $H$ consists of $\ell \geq 1$ connected components $H_{1}, \ldots, H_{\ell}$. For each component $H_{i}$ of $H$ fix a vertex $y_{i} \in V\left(H_{i}\right)$. Without loss of generality, we set $y_{1}=x_{0}$, where $x_{0}$ is the chosen vertex in the Kainen basis. We denote this set of fixed vertices by $W_{\text {fix }}$. The quadrangles of $\mathcal{K}_{a b}$ are of the form $\left\langle\left(a, x_{0}\right)\left(b, x_{0}\right)\left(a, y_{i}\right)\left(b, y_{j}\right)\right\rangle$ with $y_{i}, y_{j} \in W_{\text {fix }} . \mathcal{G}_{a}$ is defined as the set $\left\{C_{b c}^{v} \mid c \in N(a) \backslash\{b\}, v \in W_{\text {fix }} \backslash\left\{x_{0}\right\}\right\}$. 
Let $F$ be a maximal spanning forest (w.r.t. the number of edges it contains) of $H$. We now define an arbitrary orientation on all edges of $G$, so that $\{a, b\}$ is oriented from $a$ to $b$. We construct the following sets of triangles.

1. For each edge $\{a, b\} \in E(G)$ we set

$$
T_{a b}=\{\langle(a, y),(b, u),(b, v)\rangle \mid y \in V(H),\{u, v\} \in E(F)\} .
$$

2. Consider all triangles of the form $\left\{\left\langle(a, u),(a, v),\left(b, y_{i}\right)\right\rangle \mid y_{i} \in W_{\text {fix }},\{u, v\} \in\right.$ $E(F)\}$. For each edge $\{a, b\} \in E(G)$ we set

$$
T_{a b}^{\prime}=\bigcup_{y_{i} \in W_{\text {fix }}}\left\{\left\langle(a, u),(a, v),\left(b, y_{i}\right)\right\rangle \mid\{u, v\} \in E(F)\right\} .
$$

3. Set $T^{\prime}=\bigcup_{a b \in E(G)}\left(T_{a b} \cup T_{a b}^{\prime}\right)$.

4. For each $a \in V(G)$ fix an edge $\{a, b\}$ and the vertex $x_{0} \in V(H)$ that is the same $x_{0}$ as for the choice of the Kainen basis. Set

$$
T_{a}=\left\{\left\langle(a, u),(a, v),\left(b, x_{0}\right)\right\rangle \mid\{u, v\} \in E(H)-E(F)\right\} .
$$

5. Set $T^{\prime \prime}=\bigcup_{a \in V(G)} T_{a}$.

6. Set $\mathcal{T}=T^{\prime} \cup T^{\prime \prime}$.

Note that for $\ell=1, F$ is a spanning tree of $H$ and $\mathcal{T}$ coincides with the triangle set of the Berger-Jaradat MCB for the case of connected $H$. Furthermore, for each $H_{i}$ all necessary triangles of MCBs of $G \circ H_{i}$ are produced by the above construction since the connected components of $F$ are spanning trees of the corresponding components of $H$, hence $\mathcal{T}_{G \circ H_{i}} \subseteq \mathcal{T}$. The linear independence of $\mathcal{T}$ follows from almost the same arguments as in the connected case:

Lemma 3.1. The set $\mathcal{T}=T^{\prime} \cup T^{\prime \prime}$ is a linearly independent set.

Proof. First, for a fixed edge $\{a, b\}$ it is easy to see that $T_{a b}$ is a linearly independent set, since all triangles $\langle(a, y),(b, u),(b, v)\rangle$ contain different edges $\{(b, u),(b, v)\}, u, v \in$ $E(F)$ for a fixed $y \in V(H)$. All triangles that contain the same edge $\{(b, u),(b, v)\}$ are connected to different $y \in V(H)$. We therefore cannot generate a triangle $\langle(a, x),(b, u)$, $(b, v)\rangle \in T_{a b}$ from triangles $\langle(a, y),(b, u),(b, v)\rangle \in T_{a b}$. The reason is that there are no elements in $T_{a b}$ that could shift $(a, y)$ to $(a, x)$, since this would require cycles with edges in the $H^{a}$-layer. Analogously, one shows that the triangles $\left\langle(a, u),(a, v),\left(b, y_{i}\right)\right\rangle$ contained in $T_{a b}^{\prime}$ are linearly independent. $T_{a}$ is a set of linearly independent circuits because every triangle contains an edge $\{(a, u),(a, v)\}$ with $\{u, v\} \in E(H)-E(F)$ that belongs to no other triangle in $T_{a}$.

Next, we demonstrate that for a fixed edge $\{a, b\} \in E(G)$ the set $T_{a b} \cup T_{a b}^{\prime} \cup T_{a} \cup T_{b}$ is linearly independent.

First, we check that $T_{a b} \cup T_{a b}^{\prime}$ is linearly independent. To see this, notice that each $C \in$ $\operatorname{span}\left(T_{a b}\right)$ contains an edge $\{b u, b v\}$ or at least two vertices in one connected component $H_{i}^{b}$ of the $H^{b}$-layer, but every cycle in $\operatorname{span}\left(T_{a b}^{\prime}\right)$ contains at most one vertex in this $H_{i}^{b}$, because $y_{i}$ is fixed. 
Second, $T_{a b} \cup T_{a b}^{\prime} \cup T_{a}$ is linearly independent, since every triangle contained in $T_{a}$ contains edges $\{(a, u),(a, v)\}$ with $\{u, v\} \in E(H)-E(F)$ that are by construction not contained in any triangle of $T_{a b} \cup T_{a b}^{\prime}$.

Third, $T_{a b} \cup T_{a b}^{\prime} \cup T_{a} \cup T_{b}$ is linearly independent, since each triangle contained in $T_{b}$ contains edges $\{(b, u),(b, v)\}$ with $\{u, v\} \in E(H)-E(F)$ that are by construction not contained in any triangle of $T_{a b} \cup T_{a b}^{\prime} \cup T_{a}$.

Finally, $\mathcal{T}=\bigcup_{a b \in G}\left(T_{a b} \cup T_{a b}^{\prime} \cup T_{a} \cup T_{b}\right)$ is linearly independent. To see this, notice that each triangle in $T_{a b} \cup T_{a b}^{\prime} \cup T_{a} \cup T_{b}$ for a fixed edge $\{a, b\} \in E(G)$ is a triangle in $a b \circ H$. Moreover, any nontrivial linear combination of triangles in $T_{a b} \cup T_{a b}^{\prime} \cup T_{a} \cup T_{b}$ contains an edge $\{(a, x),(b, y)\}$ that cannot be cancelled by any triangles in $T_{a^{\prime} b^{\prime}} \cup T_{a^{\prime} b^{\prime}}^{\prime} \cup T_{a^{\prime}} \cup T_{b^{\prime}}$ with $\left(a^{\prime}, b^{\prime}\right) \neq(a, b)$. Thus, $\mathcal{T}=T^{\prime} \cup T^{\prime \prime}$ is a linearly independent set.

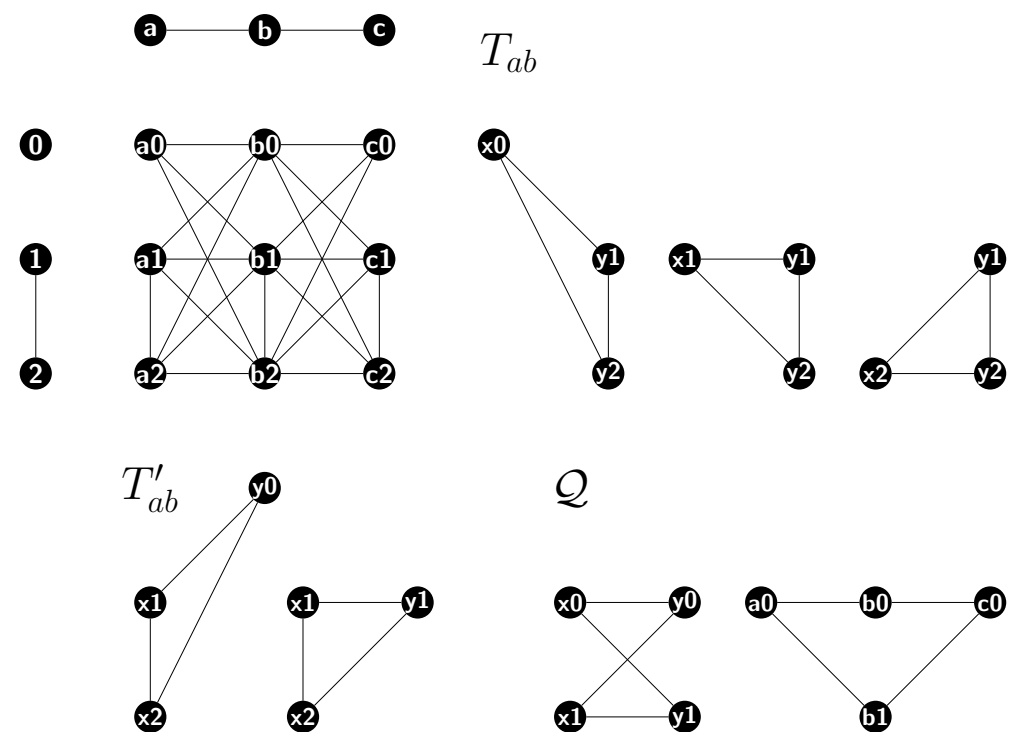

Figure 1: An MCB of the lexicographic product depicted in the upper left part. In this example we have $\{x, y\} \in\{\{a, b\},\{b, c\}\}, T^{\prime \prime}=\emptyset$, and $B^{x_{0}}=\emptyset$.

We will show in the following that the union of $\mathcal{T}, \mathcal{Q}$ and a copy of an MCB of $G$ that is located in a $G^{x_{0}}$-layer is an MCB of $G \circ H$ if $G$ is triangle-free.

Theorem 3.2. Suppose that $G \neq K_{1}$ is a connected, not necessarily triangle-free graph and $H$ has connected components $H_{i}, 1 \leq i \leq \ell$ and let $\mathcal{B}$ be an MCB of $G$. Then $\mathcal{T} \cup \mathcal{Q} \cup \mathcal{B}^{x_{0}}$ is a cycle basis of $G \circ H$. 
Proof. First, we verify that $\mathcal{T} \cup \mathcal{Q} \cup \mathcal{B}^{x_{0}}$ has the required cardinality:

$$
\begin{aligned}
&\left|\mathcal{T} \cup \mathcal{Q} \cup \mathcal{B}^{x_{0}}\right|= \\
&=\underbrace{|E(G)|(|V(H)|(|V(H)|-\ell)+\ell(|V(H)|-\ell)}_{T^{\prime}}+\underbrace{|V(G)|(|E(H)|-|V(H)|+\ell)}_{\mathcal{Q}} \\
&+\underbrace{|E(G)|(\ell-1)^{2}+(\ell-1)(2|E(G)|-|V(G)|)}_{T^{\prime \prime}}+\underbrace{|E(G)|-|V(G)|+1}_{\mathcal{B}^{x_{0}}} \\
&=\left(|E(G)||V(H)|^{2}+|V(G)||E(H)|\right)-|V(G)||V(H)|+1=\beta(G \circ H)
\end{aligned}
$$

Second, we prove that $\mathcal{T} \cup \mathcal{Q}$ is linearly independent. A cycle that is in $\operatorname{span}(\mathcal{Q})$ contains no edge and at most one vertex of each $H_{i}^{a}$-layer with $a \in V(G)$. But a cycle in $\operatorname{span}(\mathcal{T})$ contains an edge or at least two vertices in an arbitrary $H_{i}^{a}$-layer. To see this, let $C_{1} \in \mathcal{T}$ be an elementary cycle that contains an edge $e$ in one $H_{i}^{a}$-layer and let $C_{2} \in \mathcal{T}$. If $e \notin E\left(C_{2}\right)$ than $C_{1} \oplus C_{2}$ is a cycle with $e$ in $H_{i}^{a}$. If $e \in E\left(C_{2}\right)$ then $C_{1} \oplus C_{2}$ has two vertices in $H_{i}^{a}$. By induction one can easily verify that every cycle $C \in \operatorname{span}(\mathcal{T})$ contains an edge or at least two vertices in an arbitrary $H_{i}^{a}$-layer. Now, we have to examine two cases:

1. $C \in \operatorname{span}(\mathcal{T})$ is a cycle that has two vertices and no edges in any $H_{j}^{v}$. Then $C_{1} \oplus C$ is a cycle with an edge $e$ in $H_{i}^{a}$.

2. $C \in \operatorname{span}(\mathcal{T})$ contains a walk in some $H_{j}^{v}$-layer. Now, the symmetric difference with elementary cycles $C^{\prime} \in \mathcal{T}$ that contain an edge of this walk results in a cycle that has at least two vertices in some $H_{k}^{w}$-layer.

Therefore, we cannot construct a cycle $C$ of $\operatorname{span}(\mathcal{T})$ by elements of $\operatorname{span}(\mathcal{Q})$ since none of its circuits contain an edge in any $H_{i}^{a}$-layer.

Furthermore, we cannot generate elements of $\operatorname{span}\left(\mathcal{B}^{x_{0}}\right)$ with elements of $\operatorname{span}(\mathcal{T} \cup \mathcal{Q})$ because a cycle in $\operatorname{span}(\mathcal{T} \cup \mathcal{Q})$ always contains an edge of the form $\left\{\left(a, x_{i}\right),\left(b, x_{j}\right)\right\}$ with $x_{i} \neq x_{j}$.

Theorem 3.3. Suppose $G \neq K_{1}$ is triangle-free, $H$ has connected components $H_{i}, 1 \leq$ $i \leq \ell$, and $\mathcal{B}$ is an $M C B$ of $G$. Then $\mathcal{T} \cup \mathcal{Q} \cup \mathcal{B}^{x_{0}}$ is an $M C B$ of $G \circ H$.

Proof. In Theorem 3.2 we proved that $\mathcal{T} \cup \mathcal{Q} \cup \mathcal{B}^{x_{0}}$ is a cycle basis of $G$. Hence, it remains to show that this basis has minimal length.

Note, we cannot construct a triangle only with elements of $\mathcal{Q}$ or $\mathcal{B}^{x_{0}}$. To see this, notice that every triangle in $G \circ H$ has to contain an edge of a connected component $H_{i}^{a}$ of the $H^{a}$-layer, since $G$ is triangle-free. Furthermore, such an edge is by construction neither contained in $\mathcal{Q}$ nor in $\mathcal{B}^{x_{0}}$. Hence, for the construction of triangles in $G \circ H$ we need the triangles contained in $\mathcal{T}$.

Assume we could generate triangles in $G \circ H$ using elements of $\mathcal{T}$ and elements of $\mathcal{Q}$ or $\mathcal{B}^{x_{0}}$.

First, we cannot construct a triangle by elements of $\operatorname{span}(\mathcal{T})$ and elements of $\operatorname{span}(\mathcal{Q})$, since the resulting cycle has always length $l \geq 4$.

Second, we cannot construct a triangle by elements of $\operatorname{span}(\mathcal{T})$ and by elements of $\operatorname{span}\left(\mathcal{B}^{x_{0}}\right)$, since the symmetric difference with cycles in $\operatorname{span}\left(\mathcal{B}^{x_{0}}\right)$ only adds, changes 
or deletes paths in the particular $G^{x_{0}}$-layer. Hence, a cycle from $\mathcal{B}^{x_{0}}$ is no generator of any triangle because the attribute of changing or deleting paths in the $G^{x_{0}}$-layer would only be interesting to generate triangles with edges in the $G^{x_{0}}$-layer, which is triangle-free. Furthermore, all triangles that have an edge in the $G^{x_{0}}$-layer are by construction already in $\mathcal{T}$.

Thus, the only way to generate triangles in $G \circ H$ is to use triangles of $\mathcal{T}$ only. Furthermore, $\beta(G \circ H)$ is the number of elements in $\mathcal{T} \cup \mathcal{Q} \cup \mathcal{B}^{x_{0}}$ which are linearly independent. Hence, $\mathcal{T} \cup \mathcal{Q} \cup \mathcal{B}^{x_{0}}$ is an $\mathrm{MCB}$ of $G \circ H$.

The construction used in Theorems 3.2 and 3.3 is an MCB only if $G$ is triangle-free. If $G$ contains triangles, however, it becomes possible to obtain a shorter basis by replacing certain quadrangles by triangles. In the following we modify our construction to accommodate this case and replace the set $\mathcal{Q}$ resulting in a new set $\mathcal{Q}^{\star}$. We do this in two separate steps.

First, we replace $\mathcal{G}_{a}$ by a suitably selected set of squares $\widetilde{\mathcal{G}}_{a}$. Then we show how some of the elements of $\widetilde{\mathcal{G}}_{a}$ and of the Kainen basis $\bigcup_{a b \in E(G)} \mathcal{K}_{a b}$ can be replaced by appropriate triangles.

Step $1\left(\mathcal{G}_{a} \rightarrow \widetilde{\mathcal{G}}_{a}\right)$.

Consider a spanning forest $F$ of the induced subgraph $\langle N(a)\rangle$ in $G$ that consists of the trees $F_{1}, \ldots, F_{\ell}, \ell \geq 1$. We define for a fixed $x_{0}$

$$
\mathcal{G}_{a}^{\prime}=\left\{\left\langle\left(u, x_{0}\right)\left(a, x_{0}\right)\left(w, x_{0}\right)(a, v)\right\rangle \mid\{u, w\} \in E(F), x_{0} \in V(H)\right\} .
$$

We have $\left|\mathcal{G}_{a}^{\prime}\right|=|E(F)|=|N(a)|-\ell=\operatorname{deg}(a)-\ell$ with $\ell \geq 1$. Furthermore $\left|\mathcal{G}_{a}\right|=$ $\operatorname{deg}(a)-1$. To obtain the required dimension we therefore need to add $\ell-1$ additional elements if we replace $\mathcal{G}_{a}$. Therefore, we define the set $\mathcal{G}_{a}^{\prime \prime}$ that will be used in addition if $\ell-1>0$ as follows: Let $b \in N(a) \cap V\left(F_{i}\right)$ be a fixed vertex for some $i=1 \ldots \ell$. Now fix for each tree $F_{j}, j \neq i$ a vertex $c_{j}$. The set $\mathcal{G}_{a}^{\prime \prime}$ is defined as the set of all squares $\left\langle\left(b, x_{0}\right)\left(a, x_{0}\right)\left(c_{j}, x_{0}\right)(a, v)\right\rangle$ with $x_{0}, v \in V(H), v \neq y$. Hence, $\left|\widetilde{\mathcal{G}}_{a}\right|=\operatorname{deg}(a)-1$. Note that $\mathcal{G}_{a}^{\prime}=\emptyset$, if $G$ does not contain any triangles. In this case $\mathcal{G}_{a}^{\prime \prime}$ and $\mathcal{G}_{a}$ coincide. Finally, we set

$$
\mathcal{Q}^{*}:=\bigcup_{a b \in E(G)} \mathcal{K}_{a b} \cup \bigcup_{a \in V(G)} \widetilde{\mathcal{G}}_{a}
$$

\section{Step 2}

Let $\{a, b\} \in E(G)$ be an edge that is contained in $k \geq 1$ triangles in $G$. Now, we fix exactly one vertex $c$ that is contained in one of those triangle $\langle a b c\rangle$ in $G$. For each such edge $\{a, b\} \in E(G)$ and this fixed vertex $c$ one replaces all Kainen basis elements $\left\langle\left(a, x_{0}\right)\left(b, x_{0}\right)\left(a, x_{i}\right)\left(b, x_{j}\right)\right\rangle \in \mathcal{K}_{a b}$ by triangles $\left\langle\left(c, x_{0}\right)\left(a, x_{i}\right)\left(b, x_{j}\right)\right\rangle$. We denote this modified set by $\mathcal{R}$.

Let $\left\langle a_{s} a_{t} a_{u}\right\rangle$ be a triangle that is contained in $G$. Replace all squares $\left\langle\left(a_{s}, x_{0}\right)\left(a_{t}, x_{0}\right)\right.$ $\left.\left(a_{u}, x_{0}\right)\left(a_{t}, x_{j}\right)\right\rangle \in \cup_{a \in V(G)} \widetilde{\mathcal{G}}_{a}$ that contain two edges of this triangle in the $G^{x_{0}}$-layer by the triangles $\left\langle\left(a_{s}, x_{0}\right)\left(a_{t}, x_{j}\right)\left(a_{u}, x_{0}\right)\right\rangle$. This modified set will be denoted by $\mathcal{S}$.

Finally, we set

$$
\mathcal{Q}^{\star}:=\mathcal{R} \cup \mathcal{S}
$$

Notice that $|\mathcal{Q}|=\left|\mathcal{Q}^{*}\right|=\left|\mathcal{Q}^{\star}\right|$. 

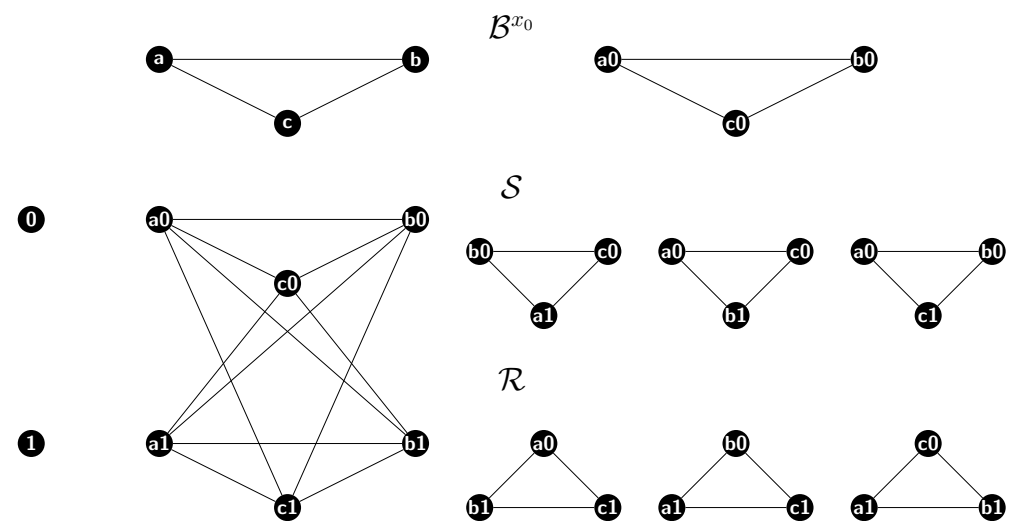

Figure 2: The MCB $\mathcal{T} \cup \mathcal{Q}^{\star} \cup \mathcal{B}^{x_{0}}$ of the lexicographic product $G \circ H$ depicted in the left part, where $\mathcal{T}=\emptyset$. The former set $\mathcal{Q}$ constructed for triangle-free factors $G$ consists of the quadrangles $\langle(a, 0)(b, 0)(a, 1)(b, 1)\rangle,\langle(a, 0)(c, 0)(a, 1)(c, 1)\rangle,\langle(b, 0)(c, 0)(b, 1)(c, 1)\rangle$, $\langle(a, 0)(c, 0)(b, 0)(b, 1)\rangle,\langle(a, 0)(b, 0)(c, 0)(b, 1)\rangle$ and $\langle(b, 0)(a, 0)(c, 0)(a, 1)\rangle$.

Theorem 3.4. Let $G \neq K_{1}$ be an arbitrary connected graph and suppose $H$ has connected components $H_{i}, 1 \leq i \leq \ell$. Furthermore, let $\mathcal{B}$ be an $M C B$ of $G$. Then $\mathcal{T} \cup \mathcal{Q}^{*} \cup \mathcal{B}^{x_{0}}$ is a cycle basis of $G \circ H$.

Proof. First, we show that all elements of the former set $\mathcal{G}_{a}$ can be generated by the elements of $\widetilde{\mathcal{G}}_{a}$.

W.l.o.g., we can choose the vertex $b \in N(a) \cap V\left(F_{i}\right)$ of $\mathcal{G}_{a}^{\prime \prime}$ as the same vertex $b$ as chosen for $\mathcal{G}_{a}$. The quadrangles of $\mathcal{G}_{a}^{\prime \prime}$ are of the form $\left\langle\left(b, x_{0}\right)\left(a, x_{0}\right)\left(c_{j}, x_{0}\right)(a, v)\right\rangle$ with $c_{j} \in F_{j}, i \neq j$. If $i=j$, i.e., the vertices $b$ and $c_{j}$ are in the same component in the maximal spanning forest, then $\left\langle\left(b, x_{0}\right)\left(a, x_{0}\right)\left(c_{j}, x_{0}\right)(a, v)\right\rangle$ is contained in $\mathcal{G}_{a}^{\prime}$.

Let $V\left(F_{j}\right)=\left\{c_{j_{1}}, \ldots, c_{j_{l}}\right\}$ and, w.l.o.g., let $c_{j}=c_{j_{1}}$ for each $F_{j}$.

If $\left|V\left(F_{j}\right)\right|=1$, then the cycle $\left\langle\left(b, x_{0}\right)\left(a, x_{0}\right)\left(c_{j}, x_{0}\right)(a, v)\right\rangle \in \mathcal{G}_{a}$ is already contained in $\mathcal{G}_{a}^{\prime \prime}$.

Let $C=\left\langle\left(b, x_{0}\right)\left(a, x_{0}\right)\left(c_{j_{t}}, x_{0}\right)(a, v)\right\rangle$ be an arbitrary cycle in $\mathcal{G}_{a}$ and assume that $\left|V\left(F_{j}\right)\right|>1$. Note, there is a unique path between the vertices $c_{j_{1}}$ and $c_{j_{t}}$ in $F_{j}$. Moreover, for any two adjacent vertices $c_{j_{s}}$ and $c_{j_{t}}$ with $s, t \leq l$ in $F_{j}$ there exists a square $\left\langle\left(c_{j_{s}}, x_{0}\right)\left(a, x_{0}\right)\left(c_{j_{t}}, x_{0}\right)(a, v)\right\rangle$ in $\mathcal{G}_{a}^{\prime}$. Therefore, we have

$$
\begin{aligned}
\left\langle\left(b, x_{0}\right)\left(a, x_{0}\right)\left(c_{j_{t}}, x_{0}\right)(a, v)\right\rangle= & \left\langle\left(b, x_{0}\right)\left(a, x_{0}\right)\left(c_{j_{1}}, x_{0}\right)(a, v)\right\rangle \\
& \oplus \bigoplus_{i=1}^{t}\left\langle\left(c_{j_{i-1}}, x_{0}\right)\left(a, x_{0}\right)\left(c_{j_{i}}, x_{0}\right)(a, v)\right\rangle .
\end{aligned}
$$

Thus, one can generate all elements of $\mathcal{G}_{a}$ by the elements of $\widetilde{\mathcal{G}}_{a}$. Moreover, since $\left|\widetilde{\mathcal{G}}_{a}\right|=$ $\left|\mathcal{G}_{a}\right|$, we conclude that $\mathcal{T} \cup \mathcal{Q}^{*} \cup \mathcal{B}^{x_{0}}$ is a cycle basis of $G \circ H$.

Theorem 3.5. Let $G \neq K_{1}$ be an arbitrary connected graph and suppose $H$ has connected components $H_{i}, 1 \leq i \leq \ell$. Furthermore, let $\mathcal{B}$ be an $M C B$ of $G$. Then $\mathcal{T} \cup \mathcal{Q}^{\star} \cup \mathcal{B}^{x_{0}}$ is an $M C B$ of $G \circ H$. 
Proof. First, we show that $\mathcal{T} \cup \mathcal{Q}^{\star} \cup \mathcal{B}^{x_{0}}$ is a basis of $G \circ H$. To this end, we show that we can generate the replaced elements of $\mathcal{Q}^{*}$ by elements of $\mathcal{Q}^{\star}$. Consider a triangle $\left\langle a_{s} a_{t} a_{u}\right\rangle$ in $G$. For a triangle $\Delta_{s, t, u}=\left\langle\left(a_{s}, x_{0}\right)\left(a_{t}, x_{i}\right)\left(a_{u}, x_{0}\right)\right\rangle \in \mathcal{S}$ in $G \circ H$ holds:

$$
\Delta_{s, t, u}=\left\langle\left(a_{s}, x_{0}\right)\left(a_{t}, x_{0}\right)\left(a_{u}, x_{0}\right)\left(a_{t}, x_{i}\right)\right\rangle \oplus\left\langle\left(a_{s}, x_{0}\right)\left(a_{t}, x_{0}\right)\left(a_{u}, x_{0}\right)\right\rangle
$$

where $\left\langle\left(a_{s}, x_{0}\right)\left(a_{t}, x_{0}\right)\left(a_{u}, x_{0}\right)\left(a_{t}, x_{i}\right)\right\rangle \in \cup_{a \in V(G)} \widetilde{\mathcal{G}}_{a} \subseteq \mathcal{Q}^{*}$. This implies that

$$
\left\langle\left(a_{s}, x_{0}\right)\left(a_{t}, x_{0}\right)\left(a_{u}, x_{0}\right)\left(a_{t}, x_{i}\right)\right\rangle=\Delta_{s, t, u} \oplus\left\langle\left(a_{s}, x_{0}\right)\left(a_{t}, x_{0}\right)\left(a_{u}, x_{0}\right)\right\rangle .
$$

If a triangle has exactly one edge in the $G^{x_{0}}$-layer and is not a basis cycle, w.l.o.g. $\left\langle\left(a_{s}, x_{0}\right)\right.$ $\left.\left(a_{t}, x_{0}\right)\left(a_{u}, x_{3}\right)\right\rangle$, then we can generate it by basis triangles.

In the course of constructing $\mathcal{S}$ we employed the forest $F$. In $F$ there is a unique path $P=\left(a_{s}, x_{0}\right)\left(b_{1}, x_{0}\right) \ldots\left(b_{p}, x_{0}\right)\left(a_{t}, x_{0}\right)$ defining triangles that have an edge in $P$ and that are connected to $\left(a_{u}, x_{3}\right)$. This leads to the following equation:

$$
\begin{aligned}
\Delta_{s, t, u}= & \left\langle\left(a_{s}, x_{0}\right)\left(a_{t}, x_{0}\right)\left(a_{u}, x_{3}\right)\right\rangle \\
= & \left\langle\left(a_{s}, x_{0}\right)\left(b_{1}, x_{0}\right)\left(b_{2}, x_{3}\right)\right\rangle \oplus \bigoplus_{i=1}^{p-1}\left\langle\left(b_{i}, x_{0}\right)\left(a_{u}, x_{3}\right)\left(b_{i+1}, x_{0}\right)\right\rangle \\
& \oplus\left\langle\left(b_{p}, x_{0}\right)\left(a_{t}, x_{0}\right)\left(a_{u}, x_{3}\right)\right\rangle \\
& \oplus\left\langle\left(a_{s}, x_{0}\right)\left(b_{1}, x_{0}\right) \ldots\left(b_{p}, x_{0}\right)\left(a_{t}, x_{0}\right)\right\rangle,
\end{aligned}
$$

where $\left\langle\left(a_{s}, x_{0}\right)\left(b_{1}, x_{0}\right) \ldots\left(b_{p}, x_{0}\right)\left(a_{t}, x_{0}\right)\right\rangle$ denotes the cycle consisting of the edges in $E(P)$ and the edge $\left\{\left(a_{s}, x_{0}\right),\left(a_{t}, x_{0}\right)\right\}$.

For a triangle $\Delta_{s, t, u}^{\prime}=\left\langle\left(a_{s}, x_{0}\right)\left(a_{t}, x_{i}\right)\left(a_{u}, x_{j}\right)\right\rangle \in \mathcal{R}$ in $G \circ H$ holds:

$$
\begin{aligned}
\Delta_{s, t, u}^{\prime}= & \left\langle\left(a_{s}, x_{0}\right)\left(a_{t}, x_{0}\right)\left(a_{u}, x_{0}\right)\left(a_{t}, x_{j}\right)\right\rangle \oplus\left\langle\left(a_{u}, x_{0}\right)\left(a_{s}, x_{0}\right)\left(a_{t}, x_{0}\right)\left(a_{u}, x_{i}\right)\right\rangle \\
& \oplus\left\langle\left(a_{s}, x_{0}\right)\left(a_{t}, x_{0}\right)\left(a_{s}, x_{i}\right)\left(a_{t}, x_{j}\right)\right\rangle \oplus\left\langle\left(a_{s}, x_{0}\right)\left(a_{t}, x_{0}\right)\left(a_{u}, x_{0}\right)\right\rangle .
\end{aligned}
$$

This implies that:

$$
\begin{aligned}
\left\langle\left(a_{s}, x_{0}\right)\left(a_{t}, x_{0}\right)(\right. & \left.\left.a_{s}, x_{i}\right)\left(a_{t}, x_{j}\right)\right\rangle= \\
= & \underbrace{\left\langle\left(a_{s}, x_{0}\right)\left(a_{t}, x_{0}\right)\left(a_{u}, x_{0}\right)\left(a_{t}, x_{j}\right)\right\rangle}_{\Delta_{s, t, u} \oplus\left\langle\left(a_{s}, x_{0}\right)\left(a_{t}, x_{0}\right)\left(a_{u}, x_{0}\right)\right\rangle} \oplus \Delta_{s, t, u}^{\prime} \oplus \\
& \underbrace{\left\langle\left(a_{u}, x_{0}\right)\left(a_{s}, x_{0}\right)\left(a_{t}, x_{0}\right)\left(a_{u}, x_{i}\right)\right\rangle}_{\Delta_{u, s, t} \oplus\left\langle\left(a_{s}, x_{0}\right)\left(a_{t}, x_{0}\right)\left(a_{u}, x_{0}\right)\right\rangle} \oplus\left\langle\left(a_{s}, x_{0}\right)\left(a_{t}, x_{0}\right)\left(a_{u}, x_{0}\right)\right\rangle \\
= & \Delta_{s, t, u}^{\prime} \oplus \Delta_{s, t, u} \oplus \Delta_{u, s, t} \oplus\left\langle\left(a_{u}, x_{0}\right)\left(a_{s}, x_{0}\right)\left(a_{t}, x_{0}\right)\right\rangle
\end{aligned}
$$

Moreover, since $\left|\mathcal{Q}^{*}\right|=\left|\mathcal{Q}^{\star}\right|$ we conclude that $\mathcal{T} \cup \mathcal{Q}^{\star} \cup \mathcal{B}^{x_{0}}$ is a cycle basis of $G \circ H$.

In order to demonstrate minimality of this basis, it remains to show that no triangle can be generated by quadrangles. By the same argument as in Theorem 3.3 we can conclude that every triangle in $a b \circ H$, where $\{a, b\} \in E(G)$ is not contained in any triangle of $G$, can only be generated by elements of $\mathcal{T}$. If $\{a, b\} \in E(G)$ is contained in a triangle of $G$, we have to show that no triangle in $a b \circ H$ can be generated by squares contained in $\mathcal{Q}^{\star} \cup \mathcal{B}^{x_{0}}$. 
We already proved that we can generate all triangles that are completely included or have an edge in one connected component $j$ of an $H^{a}$-layer by triangles in $\mathcal{T}$. Hence, it remains to prove that we can generate all triangles whose vertices are in different $H^{a}$ layers.

The following equation shows, how we can generate all triangles whose vertices are in different $H^{a}$-layers with $x_{i} \in W_{\text {fix }}$. Note, in this equation we generate triangles $\left\langle\left(a_{1}, x_{1}\right)\right.$ $\left.\left(a_{2}, x_{2}\right)\left(a_{3}, x_{3}\right)\right\rangle$ by a sum of quadrangles and one triangle. For the used quadrangles we have already shown above how one can generate them by triangles only. The triangle $\left\langle\left(a_{1}, x_{1}\right)\left(a_{2}, x_{2}\right)\left(a_{3}, x_{3}\right)\right\rangle$ can be generated by the following sum:

$$
\begin{aligned}
& \left\langle\left(a_{1}, x_{0}\right)\left(a_{2}, x_{0}\right)\left(a_{1}, x_{1}\right)\left(a_{2}, x_{2}\right)\right\rangle \oplus\left\langle\left(a_{3}, x_{0}\right)\left(a_{2}, x_{0}\right)\left(a_{3}, x_{3}\right)\left(a_{2}, x_{2}\right)\right\rangle \\
\oplus & \left\langle\left(a_{1}, x_{0}\right)\left(a_{3}, x_{0}\right)\left(a_{1}, x_{1}\right)\left(a_{3}, x_{3}\right)\right\rangle \oplus\left\langle\left(a_{1}, x_{0}\right)\left(a_{2}, x_{0}\right)\left(a_{3}, x_{0}\right)\left(a_{2}, x_{2}\right)\right\rangle \\
\oplus & \left\langle\left(a_{2}, x_{0}\right)\left(a_{3}, x_{0}\right)\left(a_{1}, x_{0}\right)\left(a_{3}, x_{3}\right)\right\rangle \oplus\left\langle\left(a_{3}, x_{0}\right)\left(a_{1}, x_{0}\right)\left(a_{2}, x_{0}\right)\left(a_{1}, x_{1}\right)\right\rangle \\
\oplus & \left\langle\left(a_{1}, x_{0}\right)\left(a_{2}, x_{0}\right)\left(a_{3}, x_{0}\right)\right\rangle
\end{aligned}
$$

In the following we show how a triangle $\left\langle\left(a_{1}, z_{1}\right)\left(a_{2}, z_{2}\right)\left(a_{3}, z_{3}\right)\right\rangle$ with $z_{i}, x_{i} \in H_{i}$ can be generated, in which $z_{i}$ must not necessarily be in $W_{\text {fix }}$. For each $z_{i}$ exists a path $P_{i}=$ $z_{i_{1}} \ldots z_{i_{p}}$ with $z_{i_{1}}=z_{i}$ and $z_{i_{p}}=x_{i} \in W_{f i x}$. As shown in the proof of Theorem 3.3, all triangles $\left\langle\left(a_{i}, z_{i_{j}}\right)\left(a_{s}, z_{s}\right)\left(a_{i}, z_{i_{k}}\right)\right\rangle$ and $\left\langle\left(a_{i}, z_{i_{j}}\right)\left(a_{t}, z_{t}\right)\left(a_{i}, z_{i_{k}}\right)\right\rangle$ and $\left\{z_{i_{j}}, z_{i_{k}}\right\} \in E\left(H_{i}\right)$ can be constructed by triangles of $\mathcal{T}$ only. Therefore, the triangle $\left\langle\left(a_{1}, z_{1}\right)\left(a_{2}, z_{2}\right)\left(a_{3}, z_{3}\right)\right\rangle$ can be generated by the following sum:

$$
\begin{aligned}
&\left\langle\left(a_{1}, x_{1}\right)\left(a_{2}, x_{2}\right)\left(a_{3}, x_{3}\right)\right\rangle \oplus \oplus \bigoplus_{\{u, v\} \in E\left(P_{1}\right)}\left(\left\langle\left(a_{1}, u\right)\left(a_{3}, x_{3}\right)\left(a_{1}, v\right)\right\rangle \oplus\left\langle\left(a_{1}, u\right)\left(a_{2}, x_{2}\right)\left(a_{1}, v\right)\right\rangle\right) \\
& \oplus \bigoplus_{\{u, v\} \in E\left(P_{2}\right)}\left(\left\langle\left(a_{2}, u\right)\left(a_{1}, z_{1}\right)\left(a_{2}, v\right)\right\rangle \oplus\left\langle\left(a_{2}, u\right)\left(a_{3}, x_{3}\right)\left(a_{2}, v\right)\right\rangle\right) \\
& \oplus \bigoplus_{\{u, v\} \in E\left(P_{3}\right)}\left(\left\langle\left(a_{3}, u\right)\left(a_{1}, z_{1}\right)\left(a_{3}, v\right)\right\rangle \oplus\left\langle\left(a_{3}, u\right)\left(a_{2}, z_{2}\right)\left(a_{3}, v\right)\right\rangle\right) .
\end{aligned}
$$

Since there is no triangle in $G \circ H$ that is generated by cycles with length $l>3$ that are contained in $\mathcal{T} \cup \mathcal{Q}^{\star} \cup \mathcal{B}^{x_{0}}$, we can conclude that $\mathcal{T} \cup \mathcal{Q}^{\star} \cup \mathcal{B}^{x_{0}}$ is an MCB.

\section{References}

[1] F Berger, Minimum cycle bases of graphs, PhD thesis, Technische Universität München, 2004.

[2] F. Berger, C. Flamm, P. M. Gleiss, J. Leydold and Peter F. Stadler, Counterexamples in chemical ring perception, J. Chem. Inf. Comput. Sci. 44 (2004), 323-331.

[3] Z. Bradshaw and M. M. M. Jaradat, Minimum cycle bases for direct products of $k_{2}$ with complete graphs, Australas. J. Comb. 43 (2009), 127-131.

[4] P. M. Gleiss, J. Leydold and P. F. Stadler, Interchangeability of relevant cycles in graphs, Elec. J. Comb. 7 (2000), R\#16, Santa Fe Institute preprint 99-07-046.

[5] R. Hammack, Minimum cycle bases of direct products of bipartite graphs, Australasian J. Comb. 36 (2006), 213-221.

[6] R. Hammack, Minimum cycle bases of direct products of complete graphs, Inf. Processing Lett. 102 (2007), 214-218. 
[7] R. Hammack and Z. Bradshaw, Minimum cycle bases of direct products of graphs with cycles, Ars Math. Contemp. 2 (2009), 101-119.

[8] R. Hammack, W. Imrich and S. Klavžar, Handbook of Product Graphs, Discrete Mathematics and its Applications, CRC Press, 2nd edition, 2011.

[9] W. Imrich and S. Klavžar, Product Graphs, Wiley-Interscience Series in Discrete Mathematics and Optimization, Wiley-Interscience, New York, 2000.

[10] W. Imrich and P. F. Stadler, Minimal cycle bases of product graphs, Australasian J. Comb. 26 (2003), 233-244.

[11] M. M. M. Jaradat, Minimal cycle bases of the lexicographic product of graphs, Discuss. Math., Graph Theory 28 (2008), 229-247.

[12] Paul C. Kainen, On robust cycle bases, Electronic Notes Discr. Math. 11 (2002), 430-437, The Ninth Quadrennial International Conference on Graph Theory, Combinatorics, Algorithms and Applications June 4-9 2000, Kalamazoo, MI.

[13] A. Kaveh and R. Mirzaie, Minimal cycle basis of graph products for the force method of frame analysis, Commun. Numer. Methods Eng. 24 (2008), 653-669.

[14] T. Kavitha, C. Liebchen, K. Mehlhorn, D. Michail, R. Rizzi, T. Ueckerdt and K. A. Zweig, Cycle bases in graphs: Characterization, algorithms, complexity, and applications, Comp. Sci. Review 3 (2009), 199-243.

[15] Ch. J. Lee, Y.-M. Kang, K.-H. Cho and K. T. Noac, A robust method for searching the smallest set of smallest rings with a path-included distance matrix, Proc Natl Acad Sci USA 106 (2009), 17355-17358.

[16] S. Lemieux and F. Major, Automated extraction and classification of RNA tertiary structure cyclic motifs, Nucleic Acids Res. 34 (2006), 2340-2346.

[17] J. Leydold and P. F. Stadler, Minimal cycle basis of outerplanar graphs, Elec. J. Comb., 5 (1998), 209-222, R\#16.

[18] C. Liebchen and R. Rizzi, Classes of cycle bases, Discr. Appl. Math. 155 (2007), 337-355. 